\title{
Fatores de Risco para Aterosclerose Associados à Aptidão Cardiorrespiratória e ao IMC em Adolescentes
}

\section{original}

\author{
Antonio Stabelini Neto \\ RODRIGO BOZZA \\ ANDERSON ZAMPIER ULBRICH \\ ÍTALO QUENNI A. DE VASCONCELOS \\ LUIS P. G. MASCARENHAS \\ MARGARET C. DA S. BOGUSZEWSKI \\ WAGNER DE CAMPOS
}

Departamento de Educação

Física e Fisioterapia da

Universidade Estadual do Norte

do Paraná (UENP), Jacarezinho,

PR, Brasil (ASN); Universidade

Federal do Paraná (UFPR),

Curitiba, PR, Brasil (MCSB, WC);

Programa de Pós-graduação

em Educação Física da UFPR,

Curitiba, PR, Brasil (ASN, RB, AZU,

IQAV, WC); Pós-graduação em

Saúde da Criança e do

Adolescente da UFPR, Curitiba,

$P R$, Brasil (LPGM, MCSB).

Recebido em 4/3/2008

Aceito em 11/5/2008

\author{
RESUMO
}

Pesquisas prévias têm demonstrado alta prevalência de fatores de risco para aterosclerose em adolescentes, no entanto, os fatores associados a seu surgimento ainda não estão totalmente esclarecidos. Portanto, este estudo teve como objetivo relacionar níveis inadequados de pressão arterial, colesterol total (CT), HDL-C, LDL-C e triglicérides (TG) com diferentes índices de $\mathrm{VO}_{2 \text { máx }}$ e índice de massa corporal (IMC) em 249 adolescentes com idades entre 12 a 16 anos. Para predição do $\mathrm{VO}_{2 \text { máx }}$ foi utilizado o teste de vai-e-vem de 20 metros. O IMC foi calculado por meio da equação: massa corporal/estatura ${ }^{2}$. Os níveis considerados inadequados foram: pressão arterial $\geq$ percentil $90^{\circ} ; \mathrm{CT} \geq$ $150 \mathrm{mg} / \mathrm{dL} ;$ LDL-C $\geq 100 \mathrm{mg} / \mathrm{dL} ; \mathrm{TG} \geq 100 \mathrm{mg} / \mathrm{dL} ;$ e HDL-C $<45 \mathrm{mg} / \mathrm{dL}$. Na análise estatística foi empregada a regressão logística, com $p<0,05$. Foram encontradas associações significativas para o sexo masculino de baixo $\mathrm{VO}_{2 \text { máx }}$ com o CT (OR 4,33; IC = 1,23-15,20) e o TG $(O R=4,88$; IC $=1,15-20,79)$ e do sobrepeso com TG $(O R=4,33$; IC =1,42-13,21). Após correção pelo IMC, os rapazes com baixos índices de $\mathrm{VO}_{2 \text { máx }}$ mantiveram as associações significativas com o $C T(O R=5,73$; IC $=1,52-21,58)$ e $T G(O R=3,81$; $I C=1,86-16,94)$. As evidências apresentadas neste estudo sugerem que, independentemente do IMC, existe relação inversa da aptidão cardiorrespiratória com o CT e o TG para os rapazes. (Arq Bras Endocrinol Metab 2008; 52/6:1024-1030)

Descritores: Aptidão cardiorrespiratória; IMC; Aterosclerose; Adolescente

\section{ABSTRACT}

\section{Atherosclerotic Risk Factors Associated with Cardiorespiratory Fitness and} BMI in Adolescents.

Previous research has demonstrated high prevalence of atherosclerotic risk factors in adolescents; however, the associate factors related to its onset are unclear. Therefore, the objective of this study was to relate inadequate blood pressure levels, total cholesterol (TC), HDL-C, LDL-C and triglycerides (TG) with different $\mathrm{VO}_{2 \text { max }}$ and $\mathrm{BMI}$ levels in a sample of 249 adolescents, aged between 12 to 16 years old. For $\mathrm{VO}_{2 \text { max }}$ prediction, the 20 meters test was used. The BMI was calculated using the body mass $/$ heigh $^{2}$ equation. The considerate inadequate levels were: blood pressure $\geq 90$ th percentile; total cholesterol $\geq$ $150 \mathrm{mg} / \mathrm{dL} ;$ LDL-C $\geq 100 \mathrm{mg} / \mathrm{dL}, \mathrm{TG} \geq 100 \mathrm{mg} / \mathrm{dL}$ and HDL-C $<45 \mathrm{mg} / \mathrm{dL}$. Logistic regression was used as statistical procedures, with $p<0.05$. For the boys, significant associations were observed between the low $\mathrm{VO}_{2 \text { máx }}$ with TC (OR 4.33; $\mathrm{IC}=1.23-15.20)$ and $\mathrm{TG}(\mathrm{OR}=4.88 ; \mathrm{IC}=1.15-20.79)$ and between overweight and $T G(O R=4.33 ; I C=1.42-13.21)$. After $B M I$ correction, the males subjects with low $\mathrm{VO}_{2 \text { max }}$ maintained their significant associations with TC $(\mathrm{OR}=5.73 ; \mathrm{IC}=1.52$ 21.58) and TG (OR=3.81; IC $=1.86-16.94)$. The evidences in this study suggested an inverse relationship of the cardiorespiratory fitness with TC and TG for boys, independently of the BMI. (Arq Bras Endocrinol Metab 2008; 52/6:1024-1030)

Keywords: Cardiorespiratory fitness; BMI; Atherosclerosis; Adolescents 


\section{INTRODUÇÃO}

A DOENÇa ARTERIal CORONARIana (DAC) é indiscutivelmente a doença mais encontrada na população brasileira, tendo seu ônus econômico crescido exponencialmente nas últimas décadas ( 1 ).

Entre os fatores de risco para aterosclerose, os mais freqüentes são a hipercolesterolemia, o tabagismo, a hipertensão arterial, a hipertrigliceridemia, a obesidade, $\mathrm{o}$ diabetes melito, o sedentarismo e a história familiar de DAC precoce $(1,2)$.

$\mathrm{O}$ exercício físico pode atuar como mecanismo preventivo, pois além da melhora da função cardiovascular, ele também promove alterações bioquímicas e hemodinâmicas significativas (redução na pressão arterial, aumento da high density lipoprotein cholesterol (HDL-C), redução da low density lipoprotein cholesterol (LDL-C) e dos triglicérides plasmáticos e aumento da tolerância à glicose), resultando melhora da saúde do indivíduo $(3,4)$.

Estudos conduzidos na população adulta têm demonstrado que a alta aptidão cardiorrespiratória está associada à redução dos riscos de $\mathrm{DAC}$, ao acidente vascular encefálico, à hipertensão arterial e ao diabetes melito $(5,6)$.

Por outro lado, diferentemente da população adulta, estudos sobre os fatores de risco para aterosclerose relacionados à aptidão cardiorrespiratória em crianças e adolescentes, além de escassos, são contraditórios (7-9).

Os pesquisadores têm especulado sobre as modificações favoráveis nas concentrações de lipídeos e lipoproteínas circulantes, teoricamente induzidas pelo exercício físico, serem confundidas pelas concomitantes alterações no peso corporal como conseqüência de alta demanda energética causada pelo exercício físico $(9,10)$.

Em vista destas considerações e pela escassez de estudos nacionais avaliando as associações da aptidão física com os fatores de risco na população pediátrica, este estudo se propôs a associar os fatores de risco para aterosclerose com os índices de aptidão cardiorrespiratória $\left(\mathrm{VO}_{2 \text { máx }}\right)$ e o índice de massa corporal (IMC) em adolescentes de ambos os sexos, de 12 a 16 anos de idade.

\section{MÉTODOS}

\section{Amostra}

A amostra aleatória simples foi composta por adolescentes de ambos os sexos, com idades entre 12 e 16 anos, matriculados na rede de ensino da cidade de São Mateus do Sul, PR, no ano letivo de 2006.

Para o cálculo do tamanho amostral, foi considerado o número de alunos matriculados por sexo e faixa etária (2.481 alunos). Estes dados foram fornecidos pela Secretaria de Educação do Município.

Em posse deste número, calculou-se a amostra, de acordo com: a) o número total de rapazes e moças; b) o IC de $95 \%$; e c) o erro amostral de $5 \%$ e a prevalência de $25 \%$ (11). Esta prevalência foi adotada, porque foi a maior encontrada em relação aos fatores de risco para aterosclerose em estudos nacionais com adolescentes (12).

Seguindo o procedimento amostral, a amostra foi estimada em 249 adolescentes, sendo 121 (48\%) rapazes e 128 (52\%) moças.

A seleção da amostra ocorreu em duas etapas. Primeiramente, das 34 escolas, foram selecionadas, de maneira não aleatória, as escolas do município que têm maior representatividade das regiões urbana e rural e que correspondem a $64,89 \%$ (1.610 alunos) do total de alunos matriculados na rede pública de ensino do município.

$\mathrm{Na}$ segunda etapa, dentro destas escolas, os indivíduos foram selecionados de maneira aleatória simples, sendo elegíveis todos os alunos com idades entre $12 \mathrm{e}$ 16 anos que atendessem a todos os critérios de inclusão para diminuir o número de variáveis intervenientes, como: a) nunca ter fumado; b) não apresentar história familiar de doença cardíaca; c) não ser diabético; d) não apresentar algum outro tipo de patologia reconhecida que possa afetar os resultados do perfil lipídico; e e) não estar ingerindo medicamento no dia do teste.

Antes do início das avaliações, os adolescentes selecionados e que concordaram em participar do estudo receberam o termo de consentimento, que foi preenchido pelos pais ou respectivos responsáveis, autorizando o uso dos seus dados. Neste termo constava breve explicação dos objetivos da pesquisa e os métodos que seriam empregados, bem como que não haveria identificação dos participantes.

Este projeto foi aprovado pelo Comitê de Ética em Pesquisa com seres humanos da Universidade Federal do Paraná, estando de acordo com a Declaração de Helsinque e com a Resolução n ${ }^{\circ}$ 196/1996.

\section{Instrumentos e procedimentos}

Todas as avaliações foram realizadas no mesmo dia, na própria escola, durante o período da manhã (das 8 às 12 horas). Foi respeitada a seguinte seqüência para a 
realização das coletas: 1) pressão arterial; 2) coleta sangüínea; 3) avaliação antropométrica; e 4) teste de aptidão cardiorrespiratória. As mensurações foram realizadas pela equipe treinada do laboratório do Centro de Pesquisa em Exercício e Esporte (CPEE), Departamento de Educação Física, Setor de Ciências Biológicas da Universidade Federal do Paraná (UFPR).

\section{Medidas antropométricas}

A massa corporal e a estatura foram mensuradas em duplicatas, sendo a média das duas medidas utilizada para calcular o IMC. Caso as medidas divergissem mais do que $0,1 \mathrm{~kg}$ e $0,1 \mathrm{~cm}$ para massa corporal e estatura, respectivamente, uma terceira medida era realizada.

A estatura total foi mensurada utilizando-se estadiômetro vertical portátil (WCS) escalonado em $0,1 \mathrm{~cm}$. Para aferir a massa corporal foi utilizada balança digital portátil (Plenna), com resolução de $100 \mathrm{~g}$. O avaliado estava descalço e vestindo somente trajes leves.

Para determinação do estado nutricional, segundo os percentis do IMC, foram utilizados os seguintes pontos de corte: eutrófico < percentil $85^{\circ}$; sobrepeso $\geq$ percentil $85^{\circ}$ e $<$ percentil $95^{\circ}$; obesidade $\geq$ percentil $95^{\circ}$. Os indivíduos foram classificados utilizando-se as tabelas de referência consoante o sexo e a faixa etária (13).

\section{Aptidão cardiorrespiratória}

Para predição do $\mathrm{VO}_{2 \text { maxx }}$, foi utilizado o teste de vai-evem de 20 metros proposto e validado por Léger e cols. (14). Este teste indireto consiste de percorrer a distância demarcada de 20 metros acompanhando determinado ritmo sonoro que define a velocidade de corrida. A velocidade aumenta em $0,5 \mathrm{~km} / \mathrm{h}$ a cada minuto, com velocidade inicial do teste de $8,5 \mathrm{~km} / \mathrm{h}$. O teste é encerrado quando o indivíduo não é mais capaz de seguir o ritmo sonoro proposto. Aplica-se a fórmula matemática que leva em consideração a idade (I) e a velocidade de corrida (V) em que foi interrompido o teste para a estimativa do consumo máximo de oxigênio. $\mathrm{VO}_{2 \max }=31.025+3.238^{*} \mathrm{~V}-3.248^{*} \mathrm{I}+0.1536^{*} \mathrm{~V}^{*} \mathrm{I}$.

Duarte e Duarte (15) demonstraram que o teste de vai-e-vem de 20 metros apresenta validade concorrente aceitável comparado com a avaliação direta do $\mathrm{VO}_{2 \text { máx }}$ recomendando sua utilização com o intuito de estimar a condição cardiorrespiratória de grupos de pessoas, principalmente em locais de pequeno espaço físico.

Os adolescentes foram classificados em quartis de acordo com os resultados alcançados no teste de apti- dão cardiorrespiratória: baixa aptidão $\mathrm{VO}_{2 \operatorname{máx}} \leq \mathrm{Ql}$, moderada $\mathrm{VO}_{2 \text { máx }}>\mathrm{Ql}$ e $<\mathrm{Q} 3$ e alta $\mathrm{VO}_{2 \text { máx }} \geq \mathrm{Q} 3$.

\section{Pressão arterial}

A pressão arterial foi mensurada pelo método auscultatório, seguindo os parâmetros estabelecidos pelo The Fourth Report on the Diagnosis, Evaluation, and Treatment of High Blood Pressure in Children and Adolescents (16). A pressão arterial sistólica (PAS) e a pressão arterial diastólica (PAD) foram mensuradas no braço direito do avaliado, após ele permanecer sentado em repouso pelo período de 10 minutos, utilizando o esfigmomanômetro de coluna de mercúrio e o estetoscópio. A PAS foi definida como o som de Korotkoff fase 1 e a PAD como o Korotkoff fase 5 .

Duas leituras foram realizadas com intervalo de 5 minutos entre as medições, sendo considerado o valor médio das duas mensurações. Caso as duas medidas diferissem mais que $4 \mathrm{mmHg}$, o protocolo era repetido.

Segundo o sexo, a idade e o percentil da estatura, foram utilizadas como ponto de corte a pressão arterial limítrofe PAS e/ou PAD $\geq$ percentil $90^{\circ}$ e a hipertensão PAS e/ou $\mathrm{PAD} \geq$ percentil $95^{\circ}$ (16).

\section{Análises do perfil lipídico}

Os indivíduos foram instruídos com uma semana de antecedência sobre alguns cuidados que deveriam tomar para participarem da coleta sangüínea, conforme recomendações da Sociedade Brasileira de Cardiologia (1), quais sejam, jejum prévio obrigatório de, no mínimo, 12 horas, evitar o consumo de álcool três dias antes do teste e evitar o abuso alimentar (em especial gordura) no dia anterior ao teste.

Para dosagem do CT, HDL-C e TG, foi usado o método enzimático-colorimétrico automatizado (Abbott Spectrum, modelo CCX). O LDL-C foi calculado pela fórmula de Friedewald (LDL-C = TC - HDL-C - TG/5) (17).

Os valores de referências que foram adotados para definir o perfil de risco aterogênico estão de acordo com a proposta apresentada para crianças e adolescentes na I Diretriz de Prevenção da Aterosclerose na Infância e Adolescência (12): CT: limítrofes 150 a $169 \mathrm{mg} / \mathrm{dL}$, aumentados $\geq 170 \mathrm{mg} / \mathrm{dL}$; colesterol HDL-C: diminuídos $<45 \mathrm{mg} / \mathrm{dL}$; colesterol LDL-C: limítrofes 100-129 mg/dL; aumentados $\geq 130 \mathrm{mg} / \mathrm{dL}$; TG: limítrofes 100 a $129 \mathrm{mg} / \mathrm{dL}$; aumentados $\geq 130 \mathrm{mg} / \mathrm{dL}$. 


\section{Tratamento dos dados e estatística}

Para o tratamento dos dados foi utilizada a estatística descritiva (média e desvio-padrão) para caracterização da amostra.

As análises de regressão logística e o intervalo de confiança (IC) de 95\%, com ajuste para a idade, foram calculados para verificar quais variáveis independentes $\left(\mathrm{VO}_{2 \operatorname{máx}}\right.$ e IMC) estavam associadas aos fatores de risco para aterosclerose (variáveis dependentes). Nestas análises, foram utilizados como referência os indivíduos com alto índice de $\mathrm{VO}_{2 \text { máx }}$ em comparação aos grupos de moderado e baixo $\mathrm{VO}_{2 \text { máx }}$. Da mesma forma para o IMC, foram considerados como referência os indivíduos com peso normal (eutróficos) em relação aos com sobrepeso e obesidade.

Os dados foram dicotomizados para cada uma das variáveis dependentes, como 0 (zero) sem fator de risco e l (um) com fator de risco para aterosclerose, sendo considerado como fator de risco os valores limítrofes e aumentados.

Os dados foram examinados no software estatístico SPSS 13.0 (Chicago, IL), com nível de significância estipulado em $\mathrm{p}<0,05$ para todas as análises.

\section{RESULTADOS}

Os dados descritivos do presente estudo para ambos os sexos são apresentados na Tabela 1. Observa-se, como esperado, maiores valores médios de estatura, massa corporal, $\mathrm{VO}_{2 \text { máx }}$ e PAS no sexo masculino em comparação com o sexo feminino.

Tabela 1. Características da amostra de adolescentes estratificadas pelo sexo.

\begin{tabular}{|c|c|c|}
\hline & $\begin{array}{c}\text { Masculino } \\
n=121\end{array}$ & $\begin{array}{c}\text { Feminino } \\
n=128\end{array}$ \\
\hline & Média DP & Média DP \\
\hline Idade (anos) & $14,95 \pm 1,30$ & $14,69 \pm 1,19$ \\
\hline Estatura (cm) & $167,24 \pm 8,33$ & $159,81 \pm 6,79$ \\
\hline Massa corporal (kg) & $56,56 \pm 10,38$ & $50,90 \pm 8,89$ \\
\hline $\operatorname{IMC}\left(\mathrm{kg} / \mathrm{m}^{2}\right)$ & $20,09 \pm 2,71$ & $19,87 \pm 2,98$ \\
\hline $\mathrm{VO}_{2 \text { máx }}\left(\mathrm{ml}^{-1} \mathrm{~kg}^{-1} \mathrm{~min}^{-1}\right)$ & $47,94 \pm 5,37$ & $40,83 \pm 4,69$ \\
\hline PAS (mmHg) & $96,64 \pm 13,76$ & $91,81 \pm 12,43$ \\
\hline PAD (mmHg) & $69,16 \pm 10,93$ & $67,89 \pm 11,53$ \\
\hline $\mathrm{CT}(\mathrm{mg} / \mathrm{dL})$ & $138,77 \pm 28,19$ & $141,58 \pm 27,18$ \\
\hline $\mathrm{HDL}-\mathrm{C}(\mathrm{mg} / \mathrm{dL})$ & $44,54 \pm 9,91$ & $47,18 \pm 14,39$ \\
\hline LDL-C (mg/dL) & $77,20 \pm 27,72$ & $77,21 \pm 26,32$ \\
\hline Triglicérides (mg/dL) & $84,90 \pm 32,60$ & $80,18 \pm 37,13$ \\
\hline
\end{tabular}

IMC = índice de massa corporal; PAS = pressão arterial sistólica; PAD = pressão arterial diastólica; CT = colesterol total; DP = desvio-padrão.

O Gráfico 1 demonstra a distribuição percentual de valores inadequados (limítrofes e aumentados) de pressão arterial, CT, LDL-C e TG e reduzidos de HDL-C, classificados conforme os valores de referência para a população pediátrica. Entre as prevalências apresentadas, a que mais chama a atenção é a alta prevalência de adolescentes com valores reduzidos de HDL-C em ambos os sexos (rapazes $60,2 \%$ e moças $49,2 \%$ ).

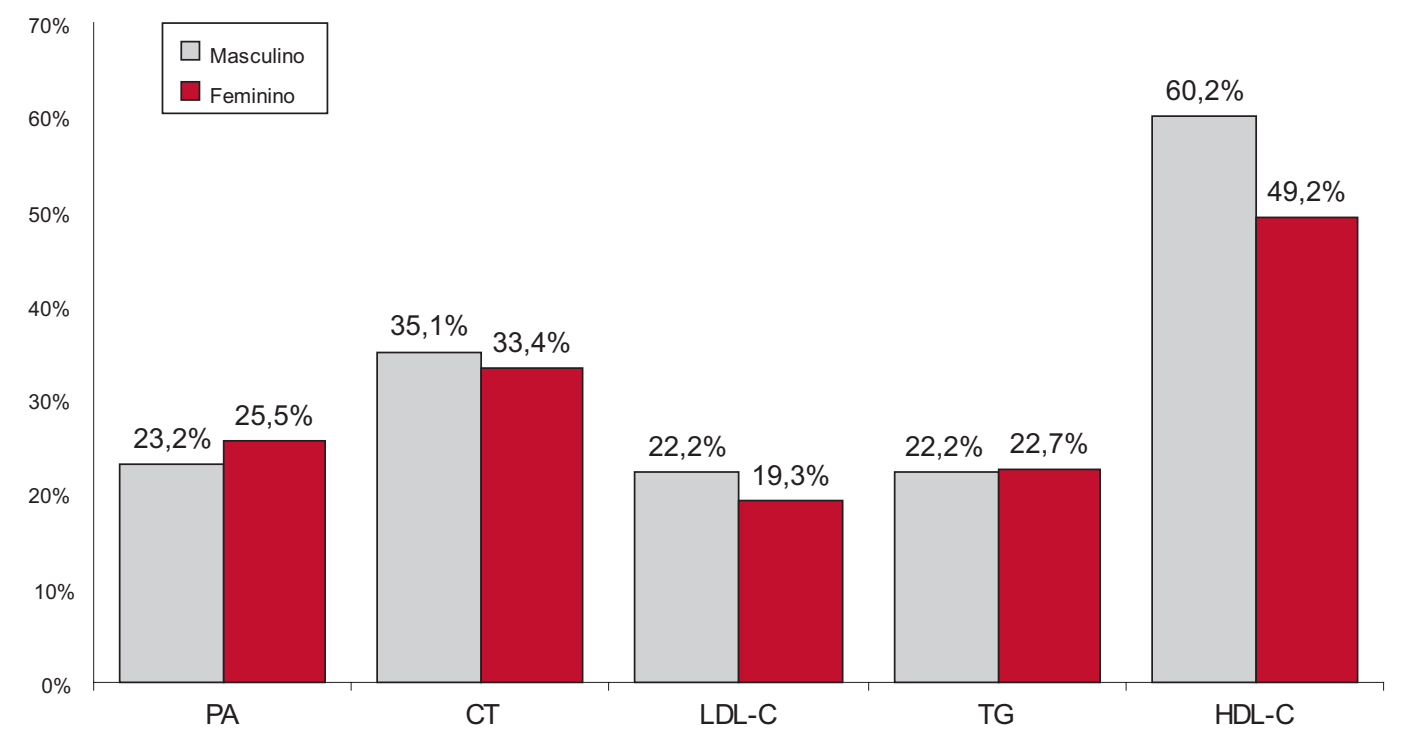

Gráfico 1. Distribuição percentual dos valores inadequados (limítrofes e aumentados) dos fatores de risco para aterosclerose. 
Mediante estimativas de odds ratio (OR) e seus respectivos IC, as associações dos índices de aptidão cardiorrespiratória e IMC com os níveis de pressão arterial, CT, lipoproteínas e triglicérides circulantes são demonstradas nas Tabelas 2 e 3, para rapazes e moças, respectivamente.

Os indivíduos do sexo masculino com baixo $\mathrm{VO}_{2 \text { máx }}$ demonstraram 4,3 vezes mais chances $(\mathrm{p}=0,02)$ de apresentar hipercolesterolemia comparados com seus pares com altos índices de $\mathrm{VO}_{2 \text { máx }}$. Da mesma forma para os TG, os indivíduos do sexo masculino com baixo $\mathrm{VO}_{2 \text { maxx }}$ apresentaram 4,8 vezes mais chances $(\mathrm{p}=0,03)$ de hipertrigliceridemia comparados com os rapazes com alto nível de aptidão cardiorrespiratória. Para as moças, não foram encontradas associações significativas dos índices de $\mathrm{VO}_{2 \text { máx }}$ com os fatores de risco para aterosclerose nas análises do presente estudo.
Considerando o IMC, os rapazes com sobrepeso apresentaram 4,3 vezes mais chances $(\mathrm{p}=0,01)$ de apresentar hipertrigliceridemia comparados com seus pares com peso normal.

Para o sexo feminino, as adolescentes com sobrepeso demonstraram quatro vezes mais chances $(\mathrm{p}=$ $0,04)$ de apresentar valores elevados de pressão arterial. Já as obesas, apresentaram 9,4 vezes mais chances $(\mathrm{p}=0,05)$ de hipertensão do que as moças com peso normal.

Ao levar em conta as significâncias estatísticas dos índices de $\mathrm{VO}_{2 \text { máx }}$ com os níveis de CT e TG observadas no sexo masculino (Tabela 2 ), optou-se por proceder à análise de regressão com ajuste do IMC para verificar o efeito isolado da aptidão cardiorrespiratória sobre os fatores de risco para aterosclerose.

Tabela 2. Associações dos índices de aptidão cardiorrespiratória e IMC com os fatores de risco para aterosclerose no sexo masculino - OR e IC.

\begin{tabular}{|c|c|c|c|c|c|}
\hline & & & RAPAZES & & \\
\hline & PA & СТ & HDL-C & LDL-C & TG \\
\hline Alto $\mathrm{VO}_{2 \text { máx }}$ & 1 & 1 & 1 & 1 & 1 \\
\hline Moderado $\mathrm{VO}_{2 \text { máx }}$ & $\begin{array}{c}1,51 \\
(0,43-5,31)\end{array}$ & $\begin{array}{c}2,00 \\
(0,64-6,25)\end{array}$ & $\begin{array}{c}0,51 \\
(0,18-1,44)\end{array}$ & $\begin{array}{c}0,74 \\
(0,21-2,56)\end{array}$ & $\begin{array}{c}2,01 \\
(0,50-8,00)\end{array}$ \\
\hline Baixo $\mathrm{VO}_{2 \text { máx }}$ & $\begin{array}{c}1,37 \\
(0,32-5,84)\end{array}$ & $\begin{array}{c}4,33^{*} \\
(1,23-15,20)\end{array}$ & $\begin{array}{c}0,58 \\
(0,17-1,90)\end{array}$ & $\begin{array}{c}1,88 \\
(0,51-6,84)\end{array}$ & $\begin{array}{c}4,88^{*} \\
(1,15-20,79)\end{array}$ \\
\hline IMC - Eutrófico & 1 & 1 & 1 & 1 & 1 \\
\hline IMC - Sobrepeso & $\begin{array}{c}0,63 \\
(0,18-2,77)\end{array}$ & $\begin{array}{c}0,63 \\
(0,20-1,97)\end{array}$ & $\begin{array}{c}2,91 \\
(0,88-9,66)\end{array}$ & $\begin{array}{c}0,16 \\
(0,02-1,34)\end{array}$ & $\begin{array}{c}4,33 \dagger \\
(1,42-13,21)\end{array}$ \\
\hline IMC - Obesidade & $\begin{array}{c}2,56 \\
(0,72-9,10)\end{array}$ & $\begin{array}{c}0,55 \\
(0,13-2,20)\end{array}$ & $\begin{array}{c}1,66 \\
(0,46-6,00)\end{array}$ & $\begin{array}{c}0,57 \\
(0,11-2,82)\end{array}$ & $\begin{array}{c}2,70 \\
(0,70-10,43)\end{array}$ \\
\hline
\end{tabular}

$\mathrm{OR}=$ odds ratio; $\mathrm{IC}=$ intervalo de confiança; ${ }^{*} \mathrm{p}<0,05 ; \uparrow \mathrm{p}<0,001 ; \mathrm{PA}=$ pressão arterial; $\mathrm{CT}=$ colesterol total; $\mathrm{TG}=$ triglicérides.

Tabela 3. Associações dos índices de aptidão cardiorrespiratória e IMC com os fatores de risco para aterosclerose no sexo feminino - OR e IC.

\begin{tabular}{|c|c|c|c|c|c|}
\hline & \multicolumn{5}{|c|}{ MOÇAS } \\
\hline & PA & CT & HDL-C & LDL-C & TG \\
\hline Alto $\mathrm{VO}_{2 \text { máx }}$ & 1 & 1 & 1 & 1 & 1 \\
\hline Moderado $\mathrm{VO}_{2 \text { máx }}$ & $\begin{array}{c}0,73 \\
(0,27-2,00)\end{array}$ & $\begin{array}{c}1,69 \\
(0,68-4,20)\end{array}$ & $\begin{array}{c}0,86 \\
(0,37-1,98)\end{array}$ & $\begin{array}{c}2,14 \\
(0,71-6,41)\end{array}$ & $\begin{array}{c}1,58 \\
(0,59-4,23)\end{array}$ \\
\hline Baixo $\mathrm{VO}_{2 \text { máx }}$ & $\begin{array}{c}2,08 \\
(0,70-6,13)\end{array}$ & $\begin{array}{c}1,14 \\
(0,38-3,41)\end{array}$ & $\begin{array}{c}0,94 \\
(0,35-2,53)\end{array}$ & $\begin{array}{c}0,40 \\
(0,07-2,23)\end{array}$ & $\begin{array}{c}0,41 \\
(0,96-1,77)\end{array}$ \\
\hline IMC - Eutrófico & 1 & 1 & 1 & 1 & 1 \\
\hline IMC - Sobrepeso & $\begin{array}{c}4,00^{*} \\
(1,06-14,99)\end{array}$ & $\begin{array}{c}1,44 \\
(0,38-5,43)\end{array}$ & $\begin{array}{c}0,69 \\
(0,18-2,57)\end{array}$ & $\begin{array}{c}1,08 \\
(0,21-5,47)\end{array}$ & $\begin{array}{c}2,50 \\
(0,65-9,57)\end{array}$ \\
\hline IMC - Obesidade & $\begin{array}{c}9,41^{*} \\
(1,74-19,0)\end{array}$ & $\begin{array}{c}2,88 \\
(0,61-13,58)\end{array}$ & $\begin{array}{c}1,38 \\
(0,29-6,45)\end{array}$ & $\begin{array}{c}1,73 \\
(0,31-9,55)\end{array}$ & $\begin{array}{c}1,50 \\
(0,27-8,21)\end{array}$ \\
\hline
\end{tabular}

$\mathrm{OR}=$ odds ratio; $\mathrm{IC}=$ intervalo de confiança; ${ }^{*} \mathrm{P}<0,05 ; \mathrm{PA}=$ pressão arterial; $\mathrm{CT}$ = colesterol total; TG = triglicérides. 
Após ajuste pelo IMC, os rapazes com baixos índices de $\mathrm{VO}_{2 \text { máx }}$ mantiveram associações estatisticamente significantes com os níveis elevados de $\mathrm{CT}(\mathrm{OR}=5,73$; IC $=1,52-21,58 ; \mathrm{p}=0,010)$ e $\mathrm{TG}(\mathrm{OR}=3,81 ; \mathrm{IC}=$ $1,86-16,94 ; \mathrm{p}=0,05)$.

\section{DISCUSSÃO}

O objetivo primário deste estudo foi analisar a associação independente entre os índices de aptidão cardiorrespiratória com os fatores de risco para aterosclerose em uma amostra aleatória de adolescentes de ambos os sexos. Os resultados deste estudo comprovaram as evidências prévias de que existe relação inversa entre os índices de $\mathrm{VO}_{2 \operatorname{máx}}$ com os fatores de risco em adolescentes do sexo masculino.

Andersen e cols. (7) avaliaram a associação da aptidão cardiorrespiratória com os fatores de risco para DAC agregados (síndrome metabólica) em adolescentes e notaram que os indivíduos com menores índices de $\mathrm{VO}_{2 \max }$ apresentavam maior número de fatores de risco do que seus pares com maiores índices de $\mathrm{VO}_{2 \text { máx }}$. Da mesma forma, Ferreira e cols. (18) observaram que, além da relação significativa com os fatores de risco, os altos índices de $\mathrm{VO}_{2 \max }$ estiveram inversamente associados com a espessura intimal da artéria carótida nos homens. Já para as mulheres, $\mathrm{o} \mathrm{VO}_{2 \text { máx }}$ não apresentou associação significativa com a espessura arterial (18).

Contrário a estes relatos, Bouziotas e cols. (8) avaliaram 210 adolescentes gregos e não encontraram qualquer tipo de associação significativa entre os índices de aptidão cardiorrespiratória com as concentrações de CT, LDL-C, HDL-C e triglicérides circulantes.

Em relação à pressão arterial, Nielsen e Andersen (9) encontraram maior razão de chances de hipertensão em adolescentes com menores índices de aptidão cardiorrespiratória, porém os achados deste estudo não suportam estas descobertas.

Quanto ao HDL-C, Stein e Stein (19) e Lima e Couto (20) relatam efeitos benéficos do exercício físico sobre os níveis circulantes em virtude da participação em diversos aspectos da atividade biológica, como o transporte reverso do colesterol, ação antioxidante, estímulo da produção de óxido nítrico e efeitos antiinflamatórios, os quais, no conjunto, resultariam proteção contra o desenvolvimento da aterosclerose. Todavia, este estudo não encontrou associação significativa entre os índices de aptidão cardiorrespiratória com os níveis de HDL-C.
Os mecanismos fisiológicos fundamentados na prática regular de exercícios físicos que levam a perfil lipídico lipoprotéico favorável ainda não são totalmente compreendidos $(19,20)$. Sugere-se que o aumento na atividade da lipase no músculo esquelético e no tecido adiposo, durante o exercício e várias horas após sua realização, associado com possível diminuição na síntese de TG no fígado podem constituir ajustamento metabólico favorável à diminuição das concentrações de lipídeos e lipoproteínas (21).

Analisando as associações do IMC com os fatores de risco hemodinâmicos para DAC, estudos prévios demonstraram relação positiva dos níveis de pressão arterial com o IMC em adolescentes de ambos os sexos (22-24). Estes relatos, em parte, foram confirmados nas análises deste estudo, já que a pressão arterial esteve significativamente associada ao sobrepeso e à obesidade para o sexo feminino, no entanto, o mesmo não ocorreu para o sexo masculino.

Apesar de a classificação dos adolescentes como hipertensos ser recurso viável e amplamente utilizado em estudos de caráter transversal, vale salientar a grande oscilação que ocorre nos valores de pressão arterial em indivíduos de idade pediátrica (16). Assim, deve-se ter cautela quanto à interpretação destes resultados.

Quanto às estimativas de OR para as associações do IMC com o perfil lipídico, observou-se que rapazes com sobrepeso têm mais chances de apresentar níveis elevados de TG. Este resultado é consistente aos achados de Freedman e cols. (23) e Kim e cols. (24), os quais, além do TG, encontraram também associações significativas do IMC com as concentrações de glicose em jejum, CT, LDL-C, HDL-C e insulina.

Assim, não obstante a inconsistência entre os estudos, especialistas da American Heart Association (25) recomendam a adoção de estilo de vida ativo associada à restrição do consumo de gorduras na dieta, principalmente do tipo saturada, como integrantes de programas para a prevenção primária de doenças ateroscleróticas em crianças e adolescentes.

Em síntese, os resultados deste estudo demonstraram que existe associação inversa entre os índices de aptidão cardiorrespiratória e os fatores de risco de aterosclerose nos indivíduos do sexo masculino, independentemente do IMC. Novas pesquisas devem ser conduzidas com caráter longitudinal, a fim de esclarecer os reais efeitos da aptidão cardiorrespiratória na promoção da saúde na população pediátrica. 
Agradecimentos: Ao Laboratório Avenida pela colaboração durante as coletas de dados, e à Secretaria Municipal de Educação, à Secretaria Municipal de Saúde e à Prefeitura Municipal da cidade de São Mateus do Sul, PR, pela disponibilidade às intituições de ensinos fundamental e médio da cidade.

Conflito de interesses: Os autores relatam não haver potencial conflito de interesses quanto ao presente artigo, e que não há conflito de interesse que possa interferir na imparcialidade deste trabaIho científico no que diz respeito às instituições mencionadas nos agradecimentos, e que estas forneceram permissão por escrito para tal.

\section{REFERÊNCIAS}

1. Sociedade Brasileira de Cardiologia. III Diretrizes Brasileiras sobre Dislipidemias e Diretriz de Prevenção da Aterosclerose do Departamento de Aterosclerose da Sociedade Brasileira de Cardiologia. Arq Bras Cardiol. 2001;77 Suppl III:1-48.

2. Gerber ZRS, Zielinsky P. Fatores de risco de aterosclerose na infância. Um estudo epidemiológico. Arq Bras Cardiol. 1997;69:231-6.

3. Boreham C, Riddoch C. The physical activity and health of children. J Sports Sci. 2001;19:915-29.

4. Twisk JWR. Physical activity guidelines for children and adolescents. Sports Med. 2001;31(8):617-27.

5. Gunnell DJ, Frankel SJ, Nanchahal K, Peters TJ, Davey-Smith G. Childhood obesity and adult cardiovascular mortality: a 57-y follow-up study based on the Boyd-Orr cohort. Am J Clin Nutr. 1998;67:1111-8.

6. Wannamethee G, Shaper AG. Physical activity and stroke in British middle-aged men. BMJ. 1992;304:597-601.

7. Andersen LB, Wedderkopp N, Hansen HS, Cooper AR, Froberg K. Biological cardiovascular risk factors cluster in Danish children and adolescents: the European Youth Heart Study. Prev Med. 2003;37:363-7.

8. Bouziotas C, Koutedakis Y, Nevill A, Ageli E, Tsigilis N, Nikolaou A, Nakou A.. Greek adolescents, fitness, fat intake, activity, and coronary heart disease risk. Arch Dis Child. 2004;89:41-4.

9. Nielsen GA, Andersen LB. The association between high blood pressure, physical fitness, and body mass index in adolescents. Prev Med. 2003;36:229-34.

10. Twisk JWR, Kemper HCG, Van Mechelen W. Tracking of activity and fitness and the relationship with cardiovascular disease risk factors. Med Sci Sports Exerc. 2000;32:1455-61.

11. Luiz RR, Magnanini MMF. A lógica da determinação do tamanho da amostra em investigações epidemiológicas. Cad Saude Publica. 2000;8(2):9-28.

12. Sociedade Brasileira de Cardiologia. I Diretriz de Prevenção da Aterosclerose na Infância e Adolescência. Arq Bras Cardiol. 2005;85:1-36

13. Kuczmarski RJ, Ogden CL, Grummer-Straw LM, et al. CDC growth charts: United States. Advance Data. 2000;314:1-27.
14. Léger LA, Mercier D, Gadoury C, Lambert J. The multistage 20 -meter shuttle run test for aerobic fitness. J Sports Sci. 1988;6:93-101.

15. Duarte MFS, Duarte CR. Validade do teste aeróbico de corrida de vai-vem de 20 metros. Rev Bras Cien e Mov. 2001;9:7-14.

16. National high blood pressure education program working group high blood pressure in children and adolescents. The Fourth Report on the Diagnosis, Evaluation, and Treatment of High Blood Pressure in Children and Adolescents. Pediatrics. 2004;114(2):555-76.

17. Friedewald WT, Levy RI, Fredrickson DS. Estimation of the concentration of low-density lipoprotein cholesterol in plasma, without use of the preparative ultracentrifuge. Clin Chem. 1972;18:499-502.

18. Ferreira I, Twisk JWR, Van Mechelen W, Kemper HCG, Stehouwer CDA. Current and adolescent levels of cardiopulmonary fitness are related to large artery properties at age 36: the Amsterdam growth and health longitudinal study. Eur J Clin Invest. 2002;32:723-31.

19. Stein O, Stein Y. Atheroprotective mechanisms of HDL. Atherosclerosis. 1999;14:285-301.

20. Lima ES, Couto RD. Estrutura, metabolismo e funções fisiológicas da lipoproteína de alta densidade. J Bras Patol Med Lab. 2006;42(3):169-78.

21. Wood PD, Stefanick ML. Exercise, fitness and atherosclerosis. In: Bouchard C, Shephard RJ, Stephens T, editores. Exercise, fitness and health: a consensus of current knowledge. Champaign: Human Kinetics Publishers; 1990. p. 409-23.

22. Ribeiro RQC, Lotufo PA, Lamounier JA, Oliveira RG, Soares JF, Botter DA. Fatores adicionais de risco cardiovascular associados ao excesso de peso em crianças e adolescentes. $O$ estudo do coração de Belo Horizonte. Arq Bras Cardiol. 2006;86(6):408-18.

23. Freedman DS, Dietz WH, Srinivasan SR, Berenson GS. The relation of overweight to cardiovascular risk factors among children and adolescents: the Bogalusa Heart Study. Pediatrics. 1999;103:1175-82.

24. Kim HM, Park J, Kim HS, Kim DH, Park SH. Obesity and cardiovascular risk factors in Korean children and adolescents aged 10-18 years from the Korean National Health and Nutrition Examination Survey, 1998 and 2001. Am J Epidemiol. 2006;1:1-7.

25. Kavey RW, Daniels SR, Lauer RM, Atkins DL, Hayman LL, Taubert K. American heart association guidelines for primary prevention of atherosclerotic cardiovascular disease beginning in childhood. Circulation. 2003;107:1561-6.

Endereço para correspondência:

Antonio Stabelini Neto

Departamento de Educação Física e Fisioterapia da UENP

Alameda Padre Magno, 841 - Caixa Postal 261

86400-000 Jacarezinho, PR

E-mail: netostabelini@hotmail.com 